4-

Bonfóronce du 15 juin 2006 organisćo par UCIODF

LA CROISSANCE
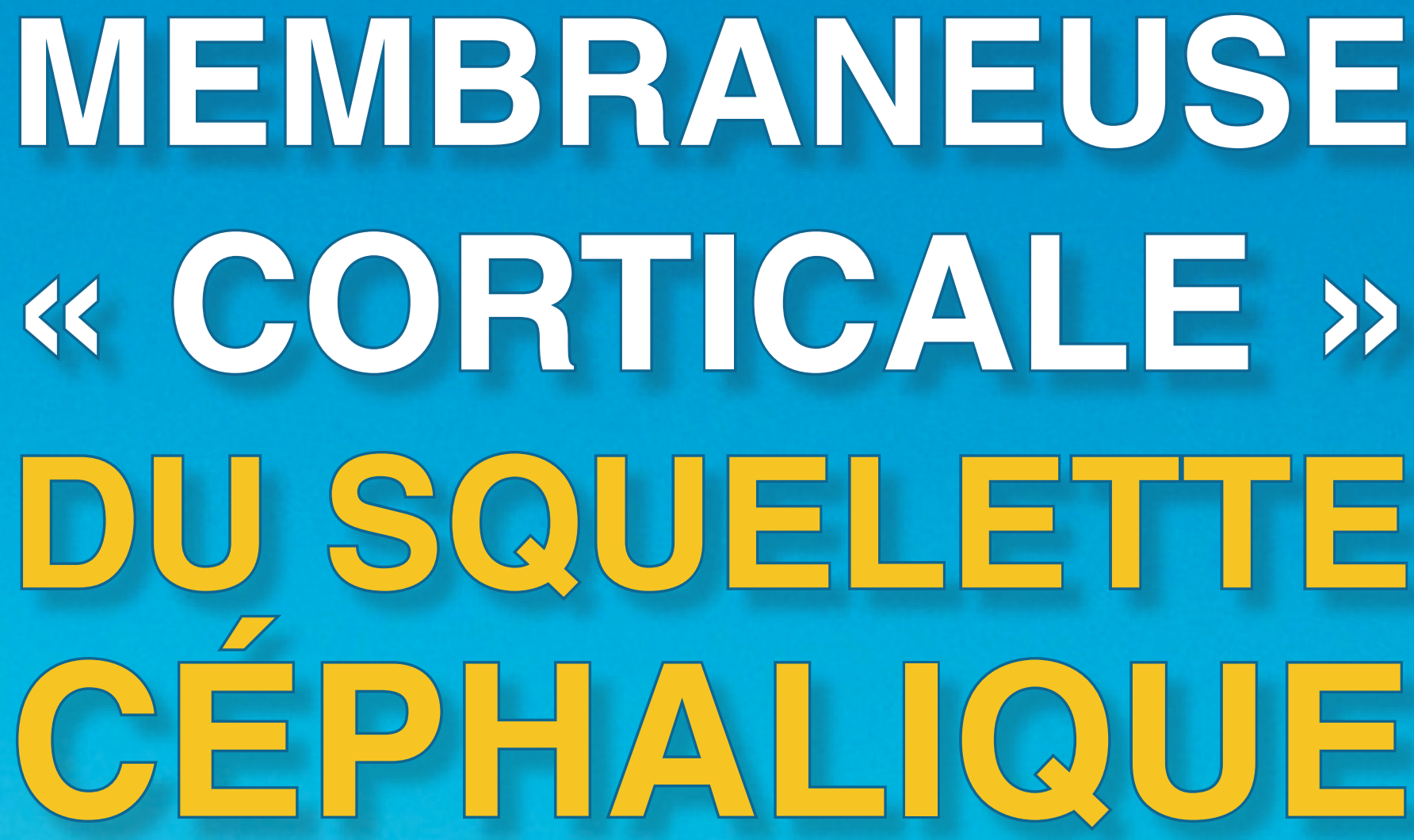

1 ère partie

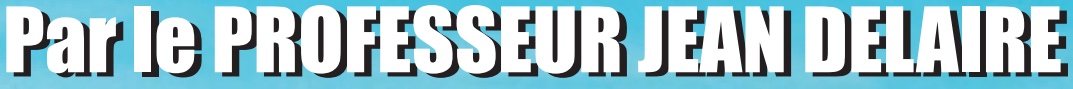

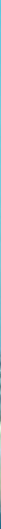




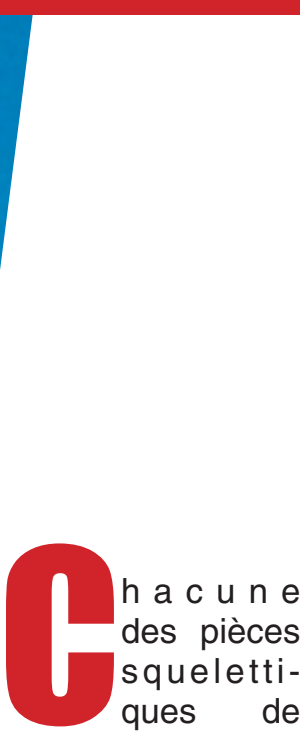

l'ensemble crânien (8 crâniennes, 13 faciales supérieures et la mandibule) possède sa croissance particulière, mais elles interfèrent toutes les unes sur les autres.

En anatomie classique on enseigne encore que :

- les différences de développement des os de la voûte, de la base du crâne et de la face, proviennent essentiellement

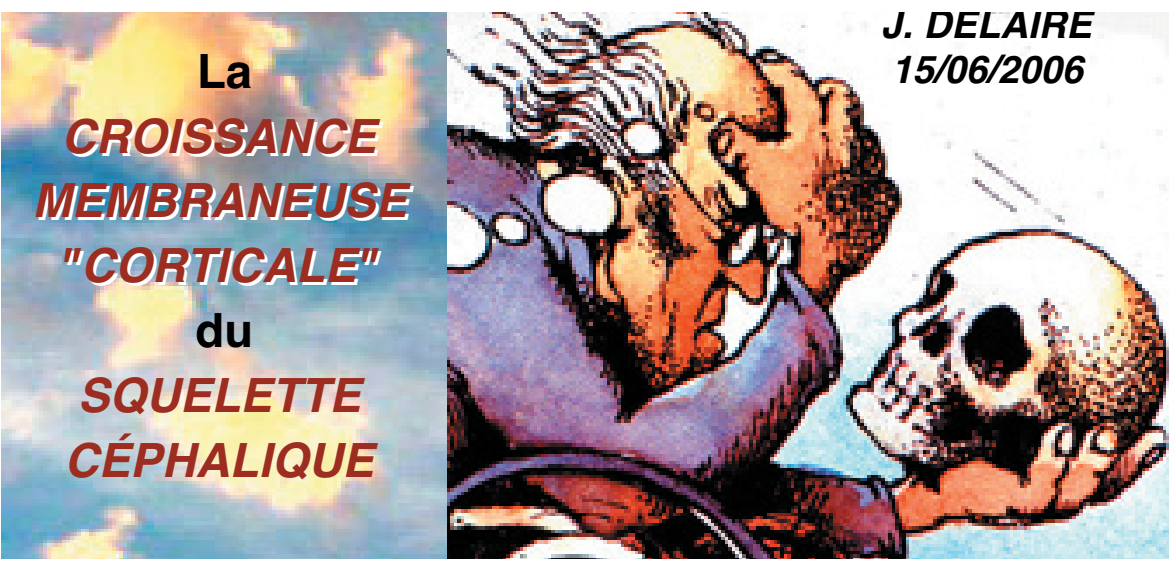

de leur origine différente (membraneuse et/ou cartilagineuse).

- la croissance interstitielle osseuse n'existe pas (SICHER)

\section{EN RÉALITÉ}

- au niveau du crâne il n'y pas véritablement de "pièce cartilagineuse ".

- toutes les pièces squelettiques céphaliques ont la même origine "Ectomésenchymateuse » (à l'exception des condyles occipitaux d'origine somitique) et s'accroissent donc, de la même façon, par les sutures qui les séparent et le périoste qui les recouvre. Les synchondroses sont des sutures " chondrifiées ». Ainsi le chondrocrâne n'a plus l'importance cartilagineuse qu'on lui donnait classiquement. C'est seulement un état cartilagineux transitoire du tissu membraneux de la base du crâne.

- D'autre part, tous les os du squelette cranio-facial :

1/ comportent plusieurs unités squelettiques ayant des modes de développement différents.

2/ il en est ainsi, notamment des corticales osseuses (qui limitent

ean DELAIRE est professeur émérite et ancien Chef de Service de Stomatologie et Chirurgie Maxillo-Faciale au Centre Hospitalier Universitaire de Nantes.

Son analyse architecturale et structurale crâniofaciale est connue et utilisée dans plusieurs pays (Angleterre, Belgique, Italie, Canada, Moyen - Orient, etc...). Elle est basée sur la recherche incessante d'équilibre ou de déséquilibre des différents constituants crânio-facio-rachidiens.

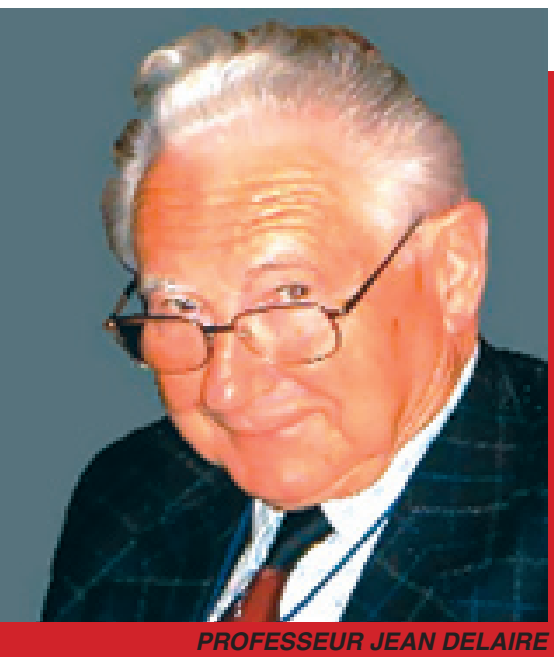

Nombreux parmi nos lecteurs connaissent et reconnaissent l'intérêt des travaux du Professeur DELAIRE à l'origine de nombreuses explications sur les phénomènes de la morphogénèse normale ou pathologique du crâne et de la face.

Nous vous présentons ici la première partie de sa conférence sur "les nouvelles données sur la croissance corticale crânio-faciale » organisée par UNIODF et qui a lieu à Nantes le 15 juin 2006. 
LES 22 PIËCES SQUELETIQUES DU SQUELETTE GRANIO-FACIAL (8 Crâniennes, 13 Faciales Supérieures + la Mandibule)

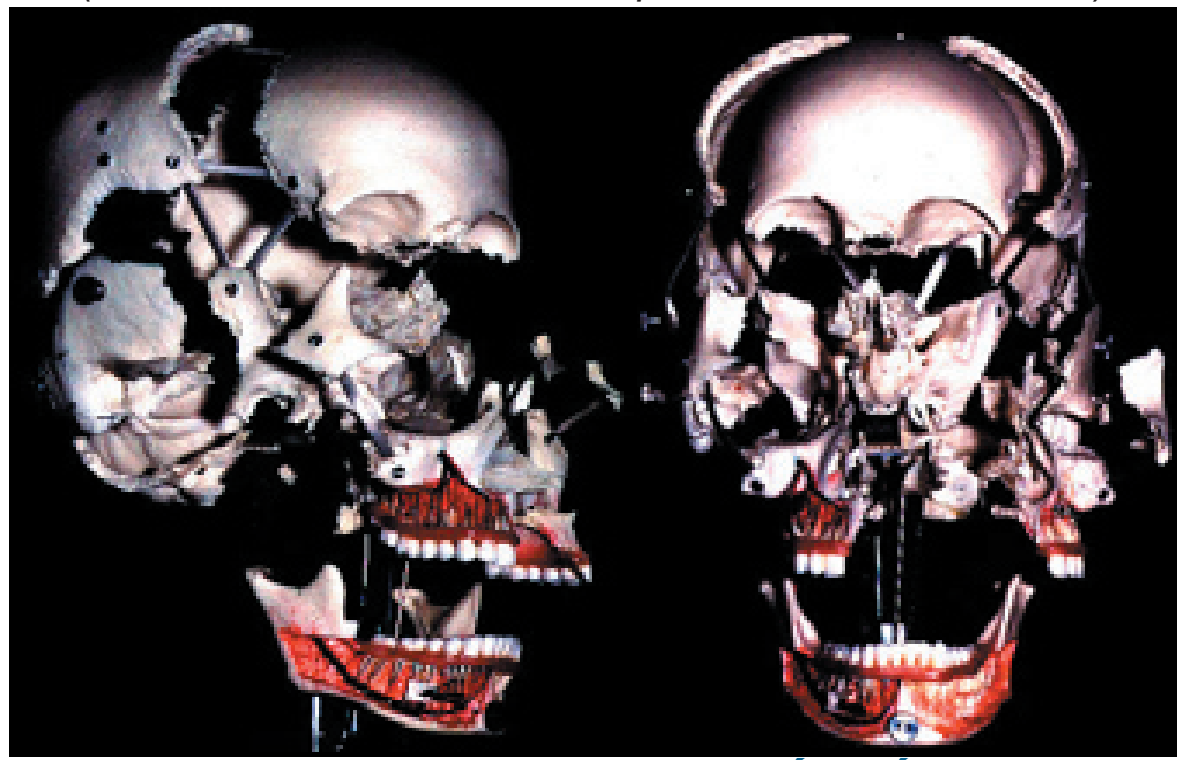

LE SQUELETTE GRANIO-FACIAL “ÉCLATÉ”
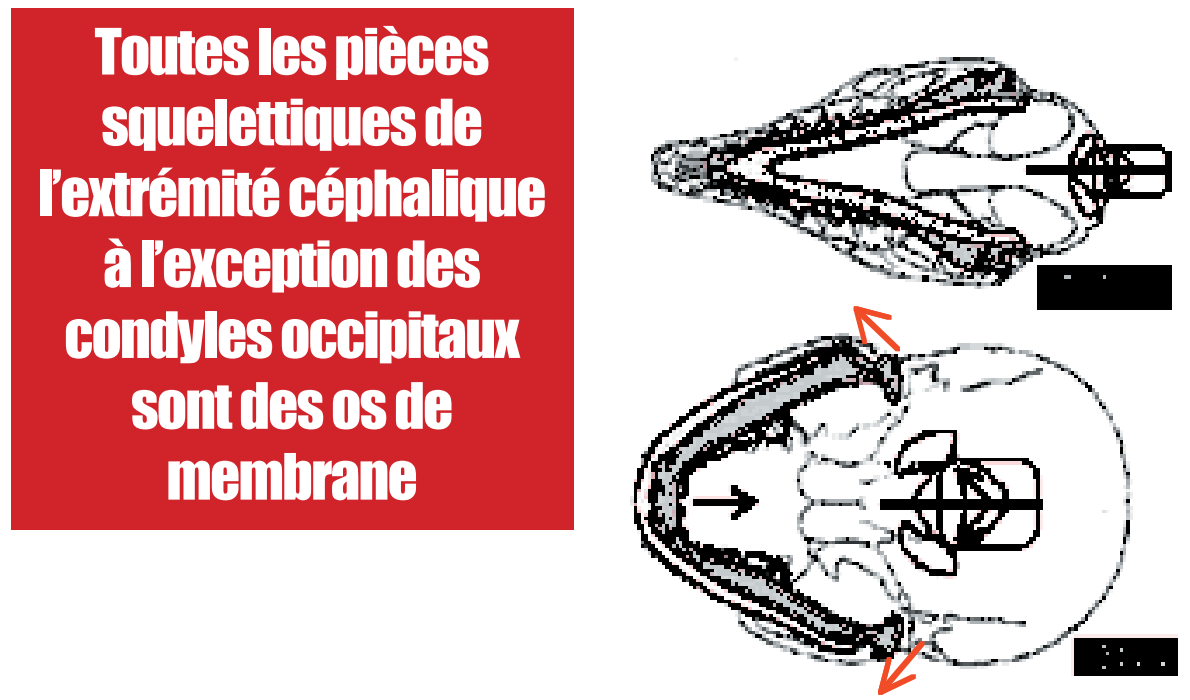

les faces superficielles et profondes de toutes les pièces squelettiques) lesquelles peuvent se déplacer excentriquement et augmenter ainsi les dimensions de l'os par un autre processus que la croissance «suturale» et les remaniements «périostés».

La conception selon laquelle la plus grande partie de la boîte crânienne (voûte et base) est formée par les cellules des crêtes neurales céphaliques et les méninges, qui en dérivent, découle des travaux de COULY et LENGELE selon lesquels seuls le basi-occipital et les exo-occipitaux aurait une origine somitique. Sachant que les expérimentations faites par COULY et vérifiées par LENGELE ont été faites chez le poulet, que les oiseaux dérivent des reptiles, et que ceux-ci ont un condyle occipital unique, il est logique de penser que l'apophyse basilaire (formée secondairement entre les deux moitiés de celui-ci) a la même origine ecto-mésenchymateuse que le corps du sphénoïde et l'écaille de l'occipital. II semble, en effet, peu vraisemblable que la méninge de la loge cérébelleuse ne puisse donner de l'os membraneux qui protège celle-ci, comme les méninges de la loge encéphalique donnent les os la voûte du crâne. Ainsi, seuls les deux condyles occipitaux seraient d'origine somitique, le reste du squelette cranio-facial: voûte, base, face, mandibule étant d'origine membraneuse.

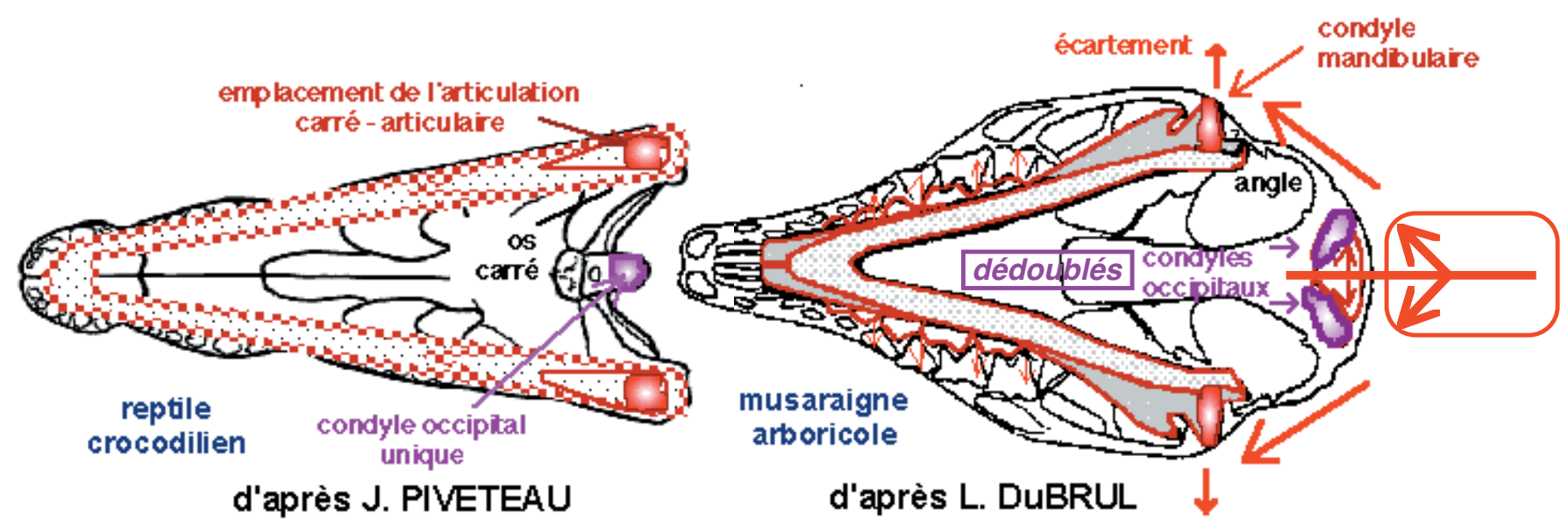


D'autre part, l'opposition classique entre le mode de croissance (secondaire) des os membraneux et celui (primaire) des os d'origine somitique est très exagérée.

En effet, la croissance «primaire » des cartilages diaphysoépiphysaires ne cesse pas toujours à la fin de la croissance (comme au niveau des os longs) mais bien souvent avant celle-ci (comme au niveau des phalanges). Après cette période, le développement des os d'origine cartilagineuse se fait aussi par des phénomènes d'apposition-résorption périostée, fondamentalement réglés par leur environnement musculaire. II en est ainsi à la base du crâne

\section{EN RÉSUMÉ}

Toutes les pièces squelettiques de l'extrémité céphalique à l'exception des condyles occipitaux sont des os de membrane. De ce fait, leur développement est essentiellement conditionné par leur environnement fonctionnel. Elles sont toutes constituées de plusieurs unités squelettiques ${ }^{1}$, chacune d'elle étant associée à une matrice fonctionnelle spécifique (MOSS). Parmi ces unités squelettiques, il y a les corticales qui peuvent évoluer d'une façon relativement indépendante les unes des autres.
Pour comprendre ce que sont exactement les unités squelettiques le mieux est de se référer à des exemples géographiques. Ainsi, tous les Etats, la France par exemple, peuvent être assimilés à des " pièces » squelettiques, délimitées par des frontières établies, comme les sutures, suite à des «tensions ", des " traumatismes ». Les unités squelettiques correspondent à leurs provinces entre lesquelles les tensions ont été moindres, ce qui a fait qu'elles ont pût s'unir en continuant pourtant à se développer sont très différemment les unes des autres. Ainsi en est-il de la Bretagne et la région Méditerranéenne (deux « unités » de la nation française) qui n'étant pas soumises aux mêmes contraintes climatiques, sociales, ethniques, évoluent bien différemment. Ces «particularités dévelopmentales individuelles » des unités squelettiques, d'une même pièce squelettique, doivent être bien connue pour pouvoir agir sélectivement sur l'une ou l'autre d'entre elles, sans avoir nécessairement besoin d'intervenir sur la totalité de la pièce.

\section{Au MAXILLAIRE :}

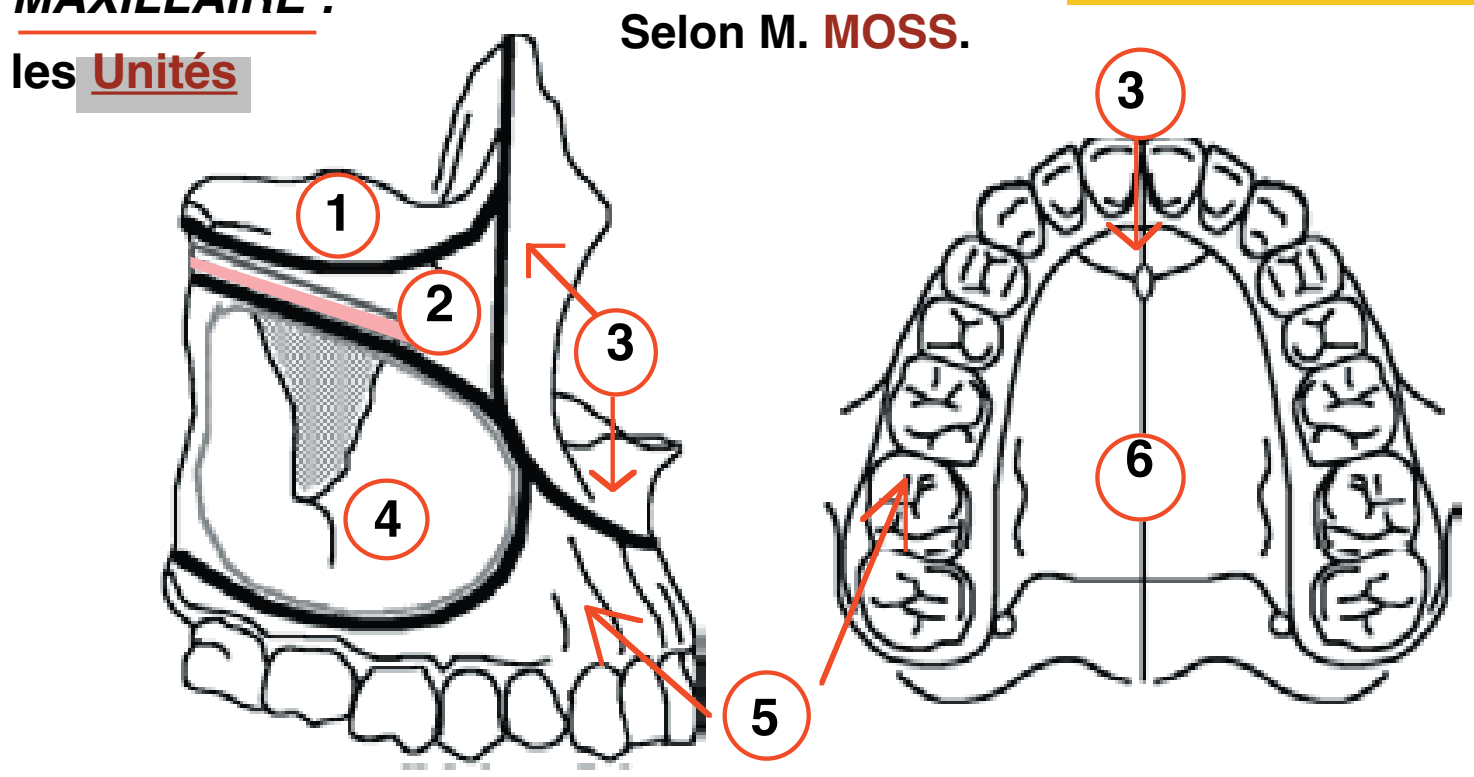

où toutes les synchondroses autres que la sphéno-occipitale (qui persiste jusqu'à l'âge adulte) se ferment avant six ans. En fait, tous les os d'origine cartilagineuse ont, aussi, un développement membraneux.

Inversement, les parties « chondrifiée » des os membraneux (exemple: le cartilage condylien et la synchondrose sphéno-occipitale) sont non seulement sensibles aux facteurs environnementaux, mais aussi aux influences générales (en particulier hormonales).

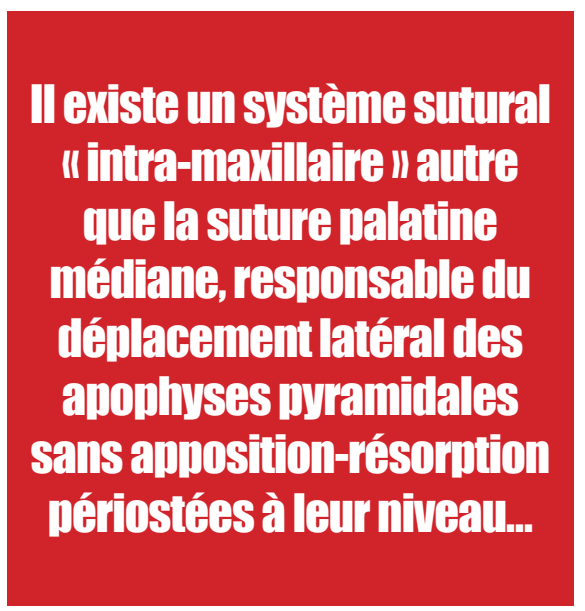

SELON M. MOSS,

le MAXILLAIRE comporterait six unités :

1- Orbitaire

2- Neurale sous-orbitaire

3- Naso-prémaxillaire

4- Sinusienne

5- Alvéolo-dentaire

6- Palatine

Ces unités, comme celles du crâne, ont des modes de développement différents sur lesquels ont peut agir différemment par nos thérapeutiques.

Selon ENLOW, la région frontale antérieure, à laquelle sont appendus 


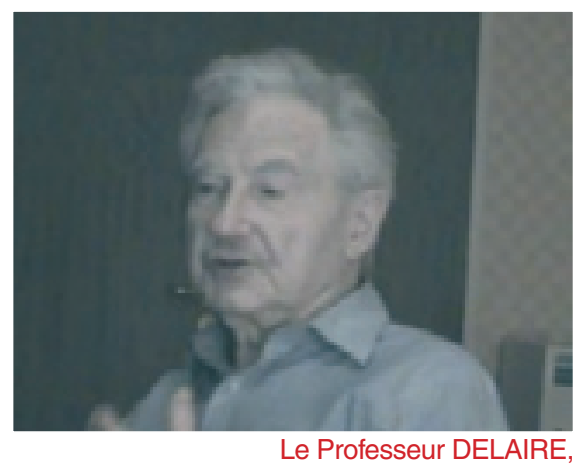

lors de la conférence du 15 juin 2006 à Nantes

Los dóplacements

ot agcroissements

sagittaux, transuersaux ot verticaux de Parcade aluéolodentaire sunǵrieuro rósultent de lactivitó fondamentalement

"I membraneuse in du périoste gingival ot des nóriostes alú́olodentaires. les deux os maxillaires supérieurs, se développerait sous l'influence exclusive de la poussée du cerveau (qui avance «en bloc» le frontal) et des appositions périostées, épaississant ses corticales antérieures (dont les parois antérieures des sinus frontaux). Le développement expansif de ces sinus résulterait des résorptions postérieures de cellesci. Ces phénomènes entraîneraient parallèlement l'avancée des parties hautes du maxillaire supérieur.

TOPINARD a proposé une autre solution que je considère personnellement comme beaucoup plus valable. Selon lui, la formation et le développement du sinus frontal résultent non pas de l'apposition corticale antérieure et de la résorption intra sinusienne, mais de l'avancée sélective de cette corticale externe par rapport à la corticale interne qui reste précocement au contact du cerveau. Ces deux lamelles, en effet, sont relativement indépendantes et "n'obéissent pas aux mêmes influences physiologiques. C'est sur la lame externe que s'attachent les ligaments et les muscles qui la développent plus ou moins suivant que le sujet est plus ou moins robuste. Tandis que la lame interne est gouvernée par le cerveau, la lame externe est en rapport avec la vie extérieure».

DE COSTER a, de même écrit : "Le frontal est le type d'os où la lame interne et la table externe montrent le mieux leurs différences. D'abord il se forme entre les deux lames du tissu spongieux, puis un véritable sinus se crée, tapissé de muqueuse, formant une cavité. Le décollement des corticales externes du frontal se fait sous l'influence des forces de mastication transmises par les apophyses montantes maxillaires et influe le développement du sinus frontal qui en résulte »

D'autres opinions concordent avec ces conceptions, dont celles de MOSS et Young, Van der KLAAUW et LEROI-GOURHAN. Dans le règne animal, de très volumineux sinus frontaux sont ainsi observés dans les espèces animales à forte mastication (hyènes, suidés «fouisseurs ") et aussi chez toutes les bêtes «à cornes», celles-ci exerçant aussi de fortes contraintes sur les corticales frontales externes. Ce rôle des « contraintes occlusales » sur le développement et l'avancée des corticales antérieures du frontal doit être

\section{Il est Important de Comprendre que}

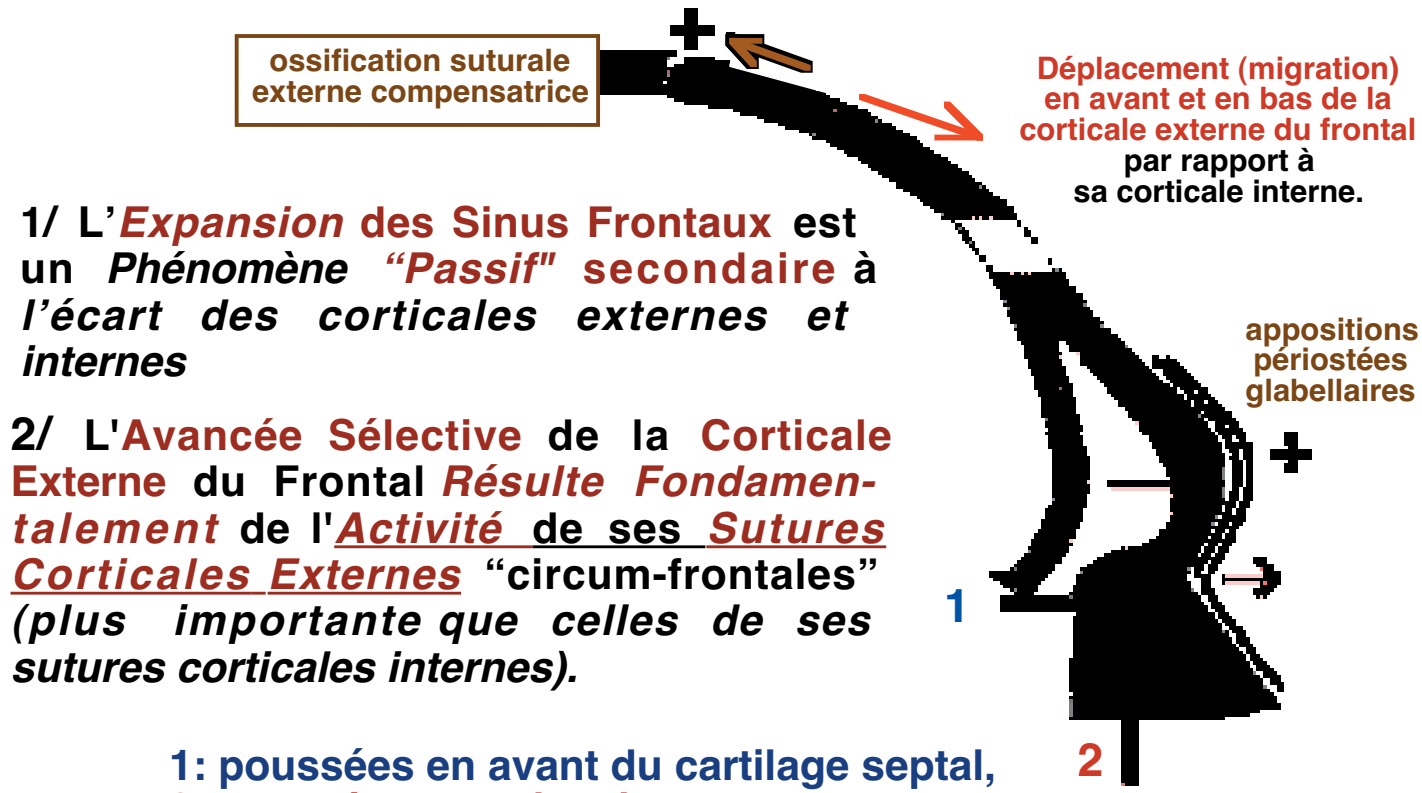

2: contraintes occlusales. 
bien connu des Orthodontistes car il conditionne directement celui des apophyses montantes maxillaires sous-jacentes.

En fait, ces déplacements corticaux de la région frontale antérieure proviennent de deux constituants anatomiques :

1- sur le plan médian, le cartilage septal qui «pousse » sur l'épine nasale antérieure du frontal, ses corticales externes, et aussi sur les os propres du nez.

2- paramédianement, les forces occlusales, qui s'exercent de part et d'autre des parties hautes des fosses nasales, par les apophyses montantes du maxillaire. Les courants piézoélectriques qui en proviennent diffusent ensuite à l'ensemble des parois antérieures de l'os frontal, contribuant notamment au développement des arcades sourcilières et de la glabelle.

Ils dépendent, d'autre part, des capacités d'apposition-résorption et de densification des cellules "ostéoprogénitrices » des sujets, variables selon les «qualités " de celles-ci, responsables aussi du «potentiel auxologique » mandibulaire. Ainsi les sujets atteints d'une maladie chromosomique, de certains syndromes malformatifs (moins sévères), ou d'une insuffisance des hormones de croissance ont-ils constamment de petits sinus. Citons, par exemple, les achondroplasies où il y peu de sinus (et inversement les acromégalies où les sinus sont très volumineux).

Ces conceptions selon lesquelles les deux corticales externes et interne des os membraneux sont relativement indépendantes et ces possibilités d'une expansion globale des pièces squelettiques ayant cette origine, ont une très grande importance en ce qui concerne la compréhension du développement du squelette cranio-facial. Elles s'appliquent, en effet, au développement de tous les os qui le constituent et, notamment, à celui du maxillaire supérieur.
$(1)$

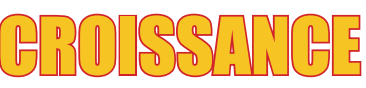
D菅

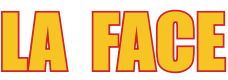

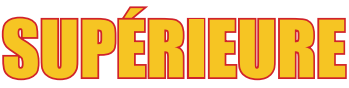
clusivement):

1/ des déplacements "en masse » du « complexe maxillaire » (et de chacune de ses pièces osseuses constitutives), avec ossification compensatrice des sutures membraneuses qui le séparent des os voisins.

2/ des appositions et résorptions périostées superficielles, contribuant à son accroissement périphérique et au modelage de ses formes externes.

\section{La Croissance Sagittale Maxillaire selon ENLOW.}

\section{2/ Par Activité Périostée :} Appositions Postérieures - Résorptions Antérieures.
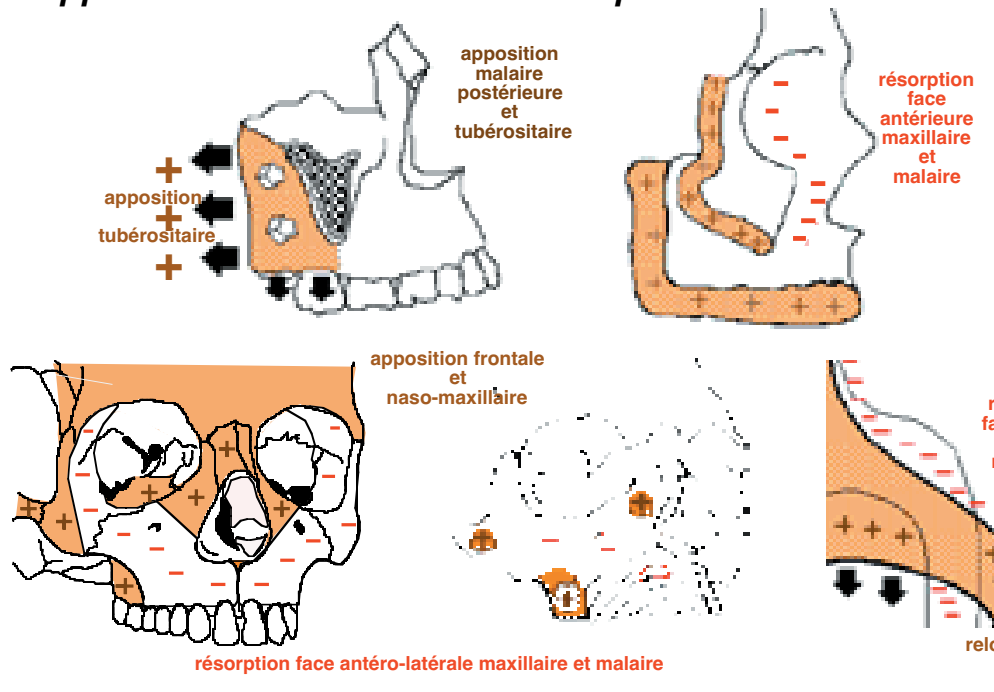

Noter que dans ce schéma d'ENLOW, l'ethmoïde de l'enfant jeune se déplacerait aussi en avant, avec l'os frontal et le maxillaire. En fait, il reste soudé au sphénoïde et ne se déplace pas. C'est ce qu'a bien montré SCOTT selon lequel, à partir de un an, la distance du foramen caecum à la selle turcique ne s'accroît plus. La totalité de l'agrandissement de la face, dans le sens sagittal, se produit dans la partie du frontal qui supporte le maxillaire sous-jacent.
De plus, le bloc maxillaire ne se déplace complètement " en masse » par rapport à la base du crâne (à part peut-être dans les premières années). Comme l'a bien dit DE COSTER, le massif maxillaire comporte d'une part un noyau central (sphénoethmoïde) très tôt immobile (fixité) et inchangé (stabilité), les seuls déplacements et accroissements squelettiques ne pouvant se produire que en avant et en dehors de lui.

II faut insister sur cette donnée essentielle : le maxillaire n'avance pas en bloc. L'avancée de ses parties antérieures est uniquement corticale. L'apposition qui s'y produit intéresse 
Tracé de H. BROADBENT (1931) Diagramme de M. HELLMAN (1932)

Noter l'importante Croissance des parties Antérieures du Maxillaire (B)
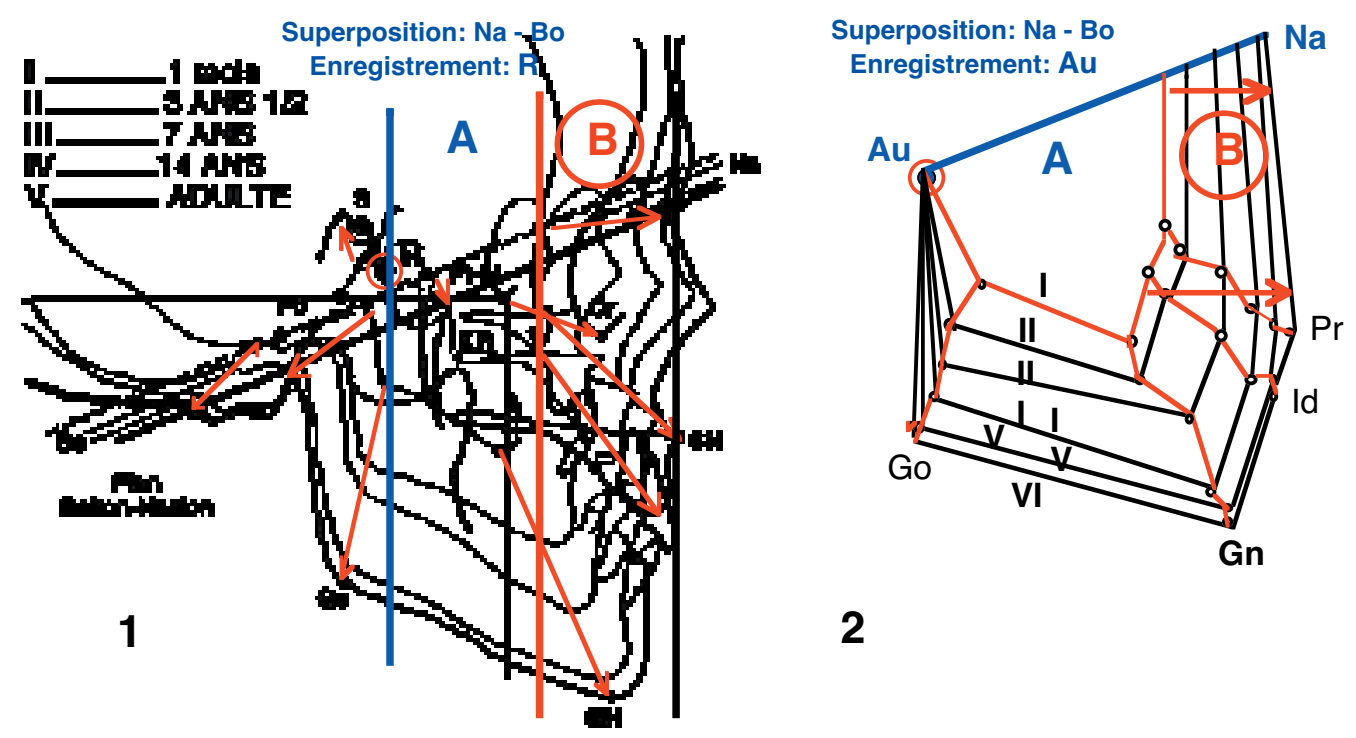

2

Les Différences de Direction de croissance obtenues par les tracés de BROADBENT et de HELLMANN sont dues à la méthode utilisée.

laire », responsable de son développement en avant de la verticale à la base du crâne passant par le foramen infra-orbitaire. Pour DE COSTER, le développement du sinus maxillaire, comme celui du sinus frontal, se produit sous l'influence des forces de mastication. Le développement « cortical » antérieur du maxillaire s'effectue ainsi (tout naturellement) en même temps que celui des parties antéro-inférieures du frontal et d'une façon manifestement coordonnée. Comme au frontal, cette avancée élective des corticales antérieures des os maxillaires supérieurs s'accompagne de leur renforcement (ceci notamment au niveau des piliers canins). Rappelons, de plus, que les dents sont fixées aux corticales inféro-externes maxillaires obligeant celles-ci à se développer pour amortir les forces qui s'y exercent. On comprend mieux, ainsi, l'importance physiologique considérable des forces «manducatrices» dans le développement (normal ou anormal) du maxillaire.

Selon BJÖRK, l'accroissement transversal du maxillaire ne pourrait provenir que de l'activité prolongée de la suture médio-palatine (dont la croissance se poursuivrait ainsi jus- qu'à l'âge adulte). Pour justifier cette opinion, il se base sur l'absence d'élimination de ses implants métalliques latéraux (placés aux parties inférieures des apophyses pyramidales maxillaires). En fait ceci prouve seulement que cette région est stable (qu'il n'y existe pas de phénomènes d'apposition-résorption) mais pas que les corticales dans lesquelles ces implants sont situés ne se déplacent pas (avec les implants qu'elles contiennent). Ses schémas montrent, de plus, que l'élargissement

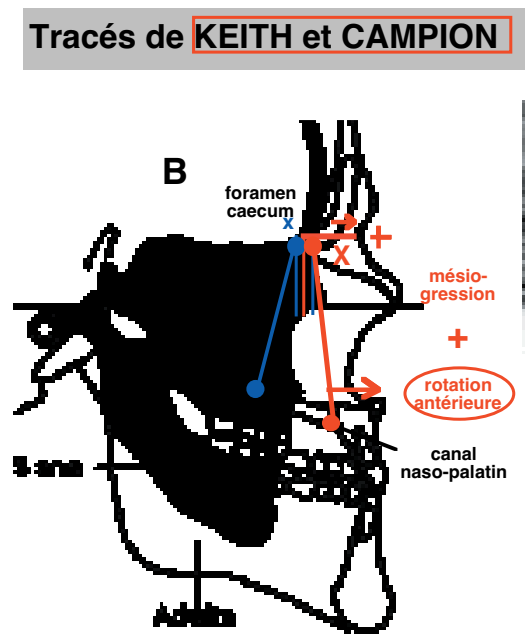

Noter I'Importance de la Croissance Antérieure et la "Rotation" Maxillaire du maxillaire est nettement plus important aux niveau de ses parties postérieures (en moyenne 7 à $9 \mathrm{~mm}$ de 4 ans à l'âge adulte) que dans ses parties antérieures (en moyenne de 1 à $2 \mathrm{~mm}$ de 10 à 20 ans). II en résulte une "rotation transversale" de chaque hémi-maxillaire, avec avancée sélective de leurs parties postérieures.

Aux conceptions de BJÖRK, opposées aux siennes, ENLOW rétorque que:

\section{SCOTT: les 2 Territoires Maxillaires.}

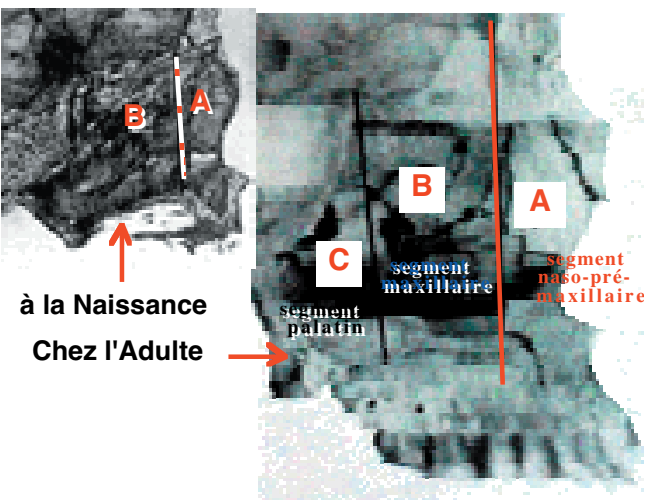

Le territoire (segment) " naso-prémaxillaire (A) situé en avant de la ligne foramen caecum canal naso-palatin) s'accroit beaucoup plu que le territoire "maxillaire" $(B)$ situé en arrièr de celle-ci. 
1/ La suture palatine médiane qui va de la partie postérieure du palais jusqu'au canal naso-palatin n'est normalement plus active après 6-7 ans.

2/ Si l'accroissement transversal du maxillaire était dû à l'élargissement de cette suture, il existerait obligatoirement une apposition osseuse (compensatrice) au niveau des parois latérales des fosses nasales (le contraire de ce qui s'y produit).

Selon lui, l'élargissement du maxillaire serait exclusivement dû à des appositions -résorptions périostées au niveau de ses parties latérales et Inférieures. Après 6-7 ans (à l'âge orthodontique), l'élargissement des fosses nasales et des orifices pyriformes résulte exclusivement de la résorption de leurs parois latérales.

La seule explication à ces divergences d'opinions de BJORK et ENLOW serait qu'il existe un système sutural autre que la suture palatine médiane, interposé entre celle-ci et les apophyses pyramidales maxillaires, responsable du déplacement latéral de celles-ci sans apposition-résorption périostées à leur niveau...

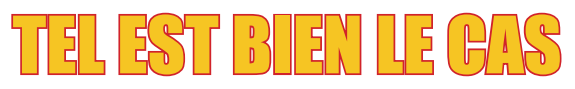

I existe, en effet, un système sutural «intra-maxillaire» décrit en Anatomie mais méconnu en Orthodontie, permettant de chaque côté les déplacements externes des corticales postéro-latérales maxillaires sans participation des fosses nasales de la suture médio-palatine. $\mathrm{Ce}$ "système » sutural est formé de chaque côté par :

1/ la suture palatine transverse (perpendiculaire à la suture palatine médiane)

$3 /$ la fissure palatine.

2/ la suture maxillo-palatine verticale

Ces six sutures permettent le déplacement bilatéral des parties postérieures du maxillaire sans élargissement des fosses nasales et sans écart des os palatins dont les tubérosités sont encastrées dans les parties inférieures des apophyses ptérygoïde.

\section{Développement des Parties Antérieures du Maxillaire}

\section{Selon DE COSTER}
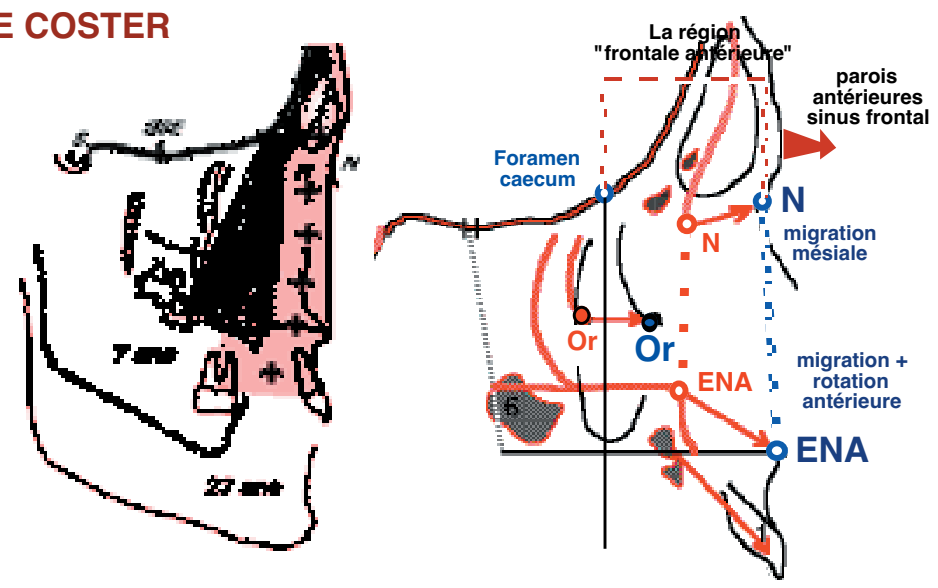

Après 7 ans, le Maxillaire s'accroit en Avant d'une Verticale abaissée à partir du Foramen Caecum.

\section{La Suture Palatine Transverse (Vues Buccale et Latérale externe) (Plus fort grossissement)}
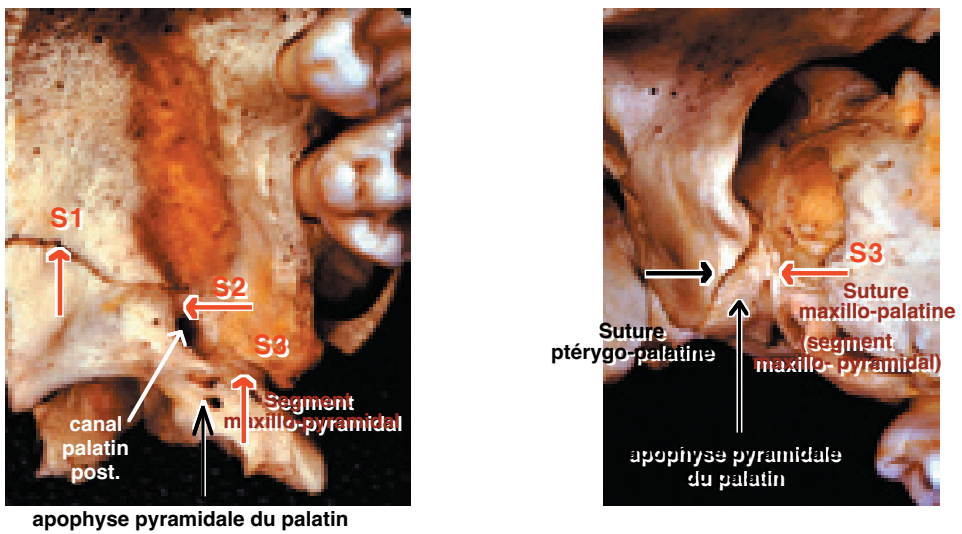

S1: segment interne transversal; S2: segment moyen oblique; S3 : segment maxillo-pyramidal.

\section{Document H. ROUVIERE.}

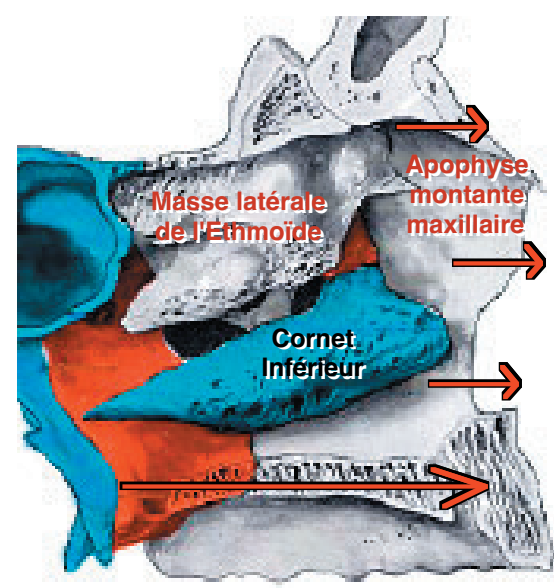

Paroi latérale des Fosses Nasales (vue Interne).
La Croissance Sagittale $\mathbf{d u}$ Maxillaire Comporte une Avancée (plus ou moins importante) des Apophyses Montantes Maxillaires, (dont les extrémités supérieures sont solidaires des corticales antérieures du frontal), par rapport aux Masses Latérales de l'Ethmoïde (qui ont atteint leur développement définitif dès la fin de la 3ème année: âge correspondant à la fin de la croissance des globes oculaires et des parties supérieures des fosses nasales). 
A partir de 7--8 ans, l'Évolution de la Dentition et les Déplacements Excentriques des Parois Sinusiennes transforment progressivement le Maxillaire en un Vaste Espace Creux circonscrit par des Corticales.

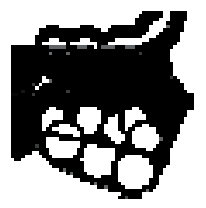

à la naissance

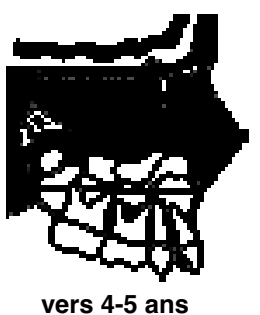

vers 4-5 ans

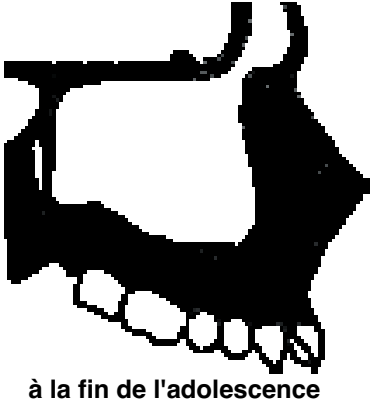

à la fin de l'adolescence
Les plus Antérieures de celles-ci s'étendent des Parois Antérieures du Frontal au Prémaxillaire, les plus Postérieures correspondent aux Tubérosités Maxillaires (alignées sur le bord antérieur des apophyses ptérygoïdes).

Ceci est très important car qui dit « suture » dit adaptabilité, «compensabilité", possibilité d'amélioration spontanée et/ou provoquée (thérapeutique) de l'état du squelette.

Notons que l'expansion (avec rotation selon BJORK) des parties tubérositaires du maxillaire entraîne aussi leur avancée. C'est donc un véritable mouvement de croissance globale du maxillaire supérieur par écartement et avancée de ses parties antéro-latérales.

Conséquences pratiques en ce qui concerne les " expansion-disjonction » maxillaires. De ce qui précède on peut déduire schématiquement que, chez le sujet de 6 à 7 ans : on peut sans difficultés majeures écarter les parois latérales des fosses nasales et des orifices pyriformes par une expansion agissant à la fois sur les sutures médio-palatine et inter-incisive médiane. Au-delà de cet âge, on ne peut que partiellement disjoindre la suture palatine médiane, bloquée par les tubérosités palatines postérieures. On peut par contre agir sur le complexe sutural décrit ci-dessus et ceci vraisemblablement jusqu'à la fin de l'adolescence (peut-être même au delà.

Résumons nous. Ce complexe sutural maxillo-palatin particulier, de part sa situation et sa souplesse donne à l'ensemble maxillaire des capacités considérables de développement cortical dans tous les sens : antéropostérieur, transversal et vertical (car la fissure palatine est oblique).
Si on remplace les triangles de BJÖRK par des courbes, on s'aperçoit, en effet, que l'avancée des parties latérales entraîne un allongement de la courbe du maxillaire donc un véritable agrandissement global.

Au total, passé les toutes premières années de la vie, le complexe sutural maxillo-palatin produit l'essentiel des accroissements sagittaux, transversaux (et en partie verticaux) du maxillaire.

Au niveau des extrémités supérieures des apophyses montantes maxillaires il se produit aussi des phénomènes particuliers. Tandis que les masses latérales de l'ethmoïde ont atteint leur développement définitif dès la fin de la $3^{\mathrm{e}}$ année (fin de croissance des globes oculaires et des parties supérieures des fosses nasales), ces apophyses montantes continuent à s'accroître et se déplacer en avant, créant ainsi entre elles et ces masses latérales un espace occupé par l'os lacrymal (unguis) dont les sutures périphériques compensent l'écart ainsi produit. ENLOW a insisté sur l'importance physiologique de ces phénomènes sans, à mon avis, les expliquer suffisamment.

\section{EN CONCLUSION,}

il faut reconsidérer la croissance du maxillaire.

Très schématiquement :

- dans les 1ères années de la vie(jusqu'à 6-7 ans) le complexe

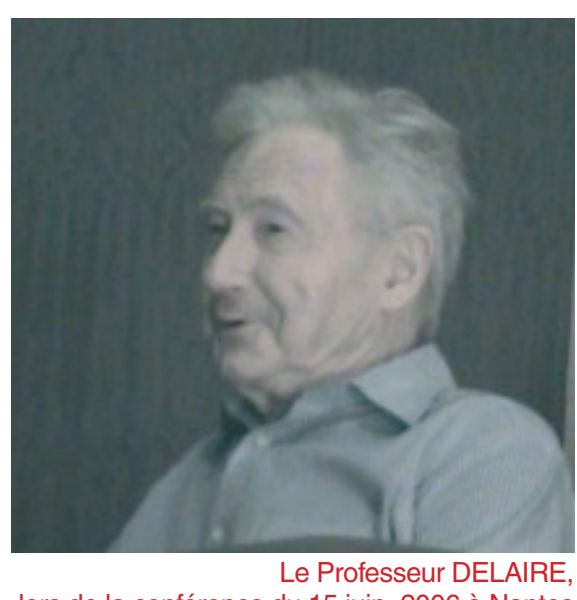

lors de la conférence du 15 juin 2006 à Nantes

maxillaire avance «en bloc", son mouvement sagittal étant normalement associé à une rotation projetant davantage en avant ses parties inférieures.

- à partir de 7 - 8 ans, le «noyau central » (DE COSTER) du maxillaire ou "centro-maxillaire » (DELAIRE), correspondant aux expansions intra faciales des os sphénoïde et ethmoïde ne se développe plus. Parallèlement, les parois latérales des fosses nasales ne se développent plus guère transversalement.

Les lames palatines, faisant partie de l'exo-péri-maxillaire cortical, continuent, par contre, à s'abaisser. De même, le naso-prémaxillaire et le prémaxillaire continuent de se développer, à s'avancer, et le sinus maxillaire s'accroît. Latéralement, les corticales qui forment leurs parois latérales se déplacent, se conforment et se renforcent sous l'influence des forces occlusales et des pressions linguales lors de la mastication et de la déglutition.

Les déplacements et accroissements sagittaux, transversaux et verticaux de l'arcade alvéolo-dentaire supérieure résultent de l'activité fondamentalement "membraneuse " du périoste gingival et des périostes alvéolo-dentaires (LEBOURG), dont l'anatomie et la physiologie sont totalement identiques à celle des autres « sutures » (SCOTT).

À SUIVRE ... 